\title{
Measurement of glass transition in native wheat flour by dynamic mechanical thermal analysis (DMTA)
}

\author{
Pedro M. Pereira ${ }^{1} \&$ Jorge C. Oliveira ${ }^{2 *}$ \\ 1 Escola Superior de Biotecnologia, Universidade Católica Portuguesa, R. Dr. António Bernardino de Almeida, Porto, \\ Portugal \\ 2 Inter-University Institute of Macau, NAPE, Lote 18, R. Londres, Edf. Tak Ip \#P, Macau
}

Summary This work describes a method to study glass transition on native starch powders, based on dynamical mechanical thermal analysis using compression tests, and was applied to wheat flour (13.5\% water content). This method will allow the determination of $\mathrm{Tg}$ in native (unprocessed) starchy materials, with minimal disturbance of the natural structures. The influence of the test conditions (heating rate, frequency and strain) on the glass transition measurements was determined using factorial designs. The values of Tg determined as the maxima of the energy dissipation (peaks in $\mathrm{E}^{\prime \prime}$ ) of native flour and of freezedried pre-gelatinized flour were not statistically different (around $64^{\circ} \mathrm{C}$ ). The heating rate did not affect the measurements in the range tested $\left(0.25\right.$ to $\left.1{ }^{\circ} \mathrm{C} \mathrm{min}{ }^{-1}\right)$. An interactive effect of the strain amplitude and the frequency was detected. The significance of this interaction can be caused by differences in mechanical energy dissipation, which would indicate that not only temperature but also the total energy input may affect this transition. Slight effects of phase separation between gluten and starch were found on native flour.

Keywords Phase transitions, starch, viscoelastic properties.

\section{Introduction}

Low moisture, amorphous or semi-crystalline biomaterials, such as flours, behave as meta-stable systems, where molecular mobility plays a major role on the kinetics of (desirable or undesirable) chemical and biochemical reactions. The state of the matrix components and its structure is therefore a key factor in the definition of the mechanical properties, stability or reactivity of a given system. Changes in molecular mobility and viscosity that take place relatively close to the state tran-

*Correspondent: SPI - Sociedade Portuguesa de Inovação Edf. Les Palaces, R. Júlio Dinis, 242 - S.208, 4050 Porto, Portugal. Fax: +351 2 6099164;

email: JMFCO@hotmail.com sition known as glass transition affect the physical stability and processability of the amorphous fractions of foods and the rate of chemical reactions (del Pilar-Buera et al., 1995). The glass transition of biopolymers is, thus, a key parameter for defining the mechanical and storage properties of foods (Kalichevsky \& Blanshard, 1992).

The glass transition of biological and food materials is a dynamic process influenced by temperature, time and composition of the matrix, whereby an amorphous matrix changes from a glassy mechanical solid (capable of supporting its own weight against flow due to the force of gravity), where molecular mobility is restricted to vibration, to a soft rubbery state, where re-crystallization, microbial growth, enzymatic activity and general degradation reactions may occur (Slade \& Levine, 
1991). The transition from a glassy to a rubbery state and the accompanying loss of stiffness, hardness, or strength are traditionally described by a relationship between the magnitudes of these parameters and temperature, moisture content, or water activity (Peleg, 1994). A material in glassy state is generally regarded as stable, while rubbery state is necessary for processability.

The glass transition, as a second order transition (a change of state but not of phase - Roos, 1995), takes place over a range of temperatures, being the midpoint of the range determined by differential scanning calorimetry (DSC) conventionally taken as the single reference point, named $\mathrm{Tg}$. However, whether Tg should be the midpoint, the onset or the endset of glass transition is a matter of discussion (Oliveira et al., 1999). The importance of the glass transition temperature range as a reference parameter for an increasing number of applications in food science and engineering involving stability and reaction kinetics in amorphous matrixes is very clear (Roos, 1995). The measurement of $\mathrm{Tg}$ for any given substance is crucial for the application of these concepts in product and process design and optimization. Available methods rely on the changes of the macroscopic material properties (such as heat capacity, thermal expansion coefficient, viscosity, elasticity, etc) associated to the changes of molecular mobility around the glass transition (Roos, 1995).

In dynamic mechanical thermal analysis (DMTA) three major changes are detected when the viscoelastic properties of the materials are analyzed against temperature: a significant drop in the storage modulus $\left(\mathrm{E}^{\prime}\right)$, which is constant in the glassy state; a peak in the loss modulus $\left(\mathrm{E}^{\prime \prime}\right)$, related to the resonance frequency of the molecular motions (Allen, 1993); and a peak in the viscoelasticity ratio ( $\tan \delta)$, which is also frequency-dependent. These changes have been correlated with, respectively, the onset, midpoint and endset of the transition as detected by DSC (MacInnes, 1993; Roos, 1995). Moreover, DMTA is a frequency-response analysis, that is, the parameters measured will show a frequency dependence which is a mirror image of the time-dependence of the state transition. While raising problems on how to compare Tg measurements between DMTA and DSC, this offers new possibilities to investigate glass transition.

Wheat flour is one of the most used food raw materials world-wide, presenting a very complex structure partially crystalline, partially amorphous, comprising a number of components that can undergo a glass transition process. Most of the relevant components have been thoroughly studied individually, as isolated substances (wheat starch Biliaderis et al., 1986; Zeleznak \& Hoseney, 1987; amylopectin - Kalichevsky et al., 1992; Kalichevsky \& Blanshard, 1993; gluten - Hoseney et al., 1986; Kalichevsky et al., 1992; glutenin - Kalichevsky et al., 1992; gliadin - Graaf et al., 1993) or as binary mixtures of the pure substances (amylose and gluten - Kalichevsky \& Blanshard, 1992). Although this approach enables a more precise insight into the behaviour of each component, the effect of the complexity of the whole structure on glass transition cannot be accurately inferred from model systems (e.g. the granular structure of flour, with crystalline and amorphous regions, the interactions between components that cannot be reproduced on artificial mixtures, the intra-granular structure). It is also noted that in most methods used to determine $\mathrm{Tg}$ in flours (DSC for example), either the sample preparation process can be disrupting in terms of the native structure and component distribution, which can potentially affect the measured value or, at low moisture contents, the transitions are not clearly defined and the methods do not have a very good accuracy.

The objective of this work was the development of a glass transition measurement method that could be applied to native flours, based on DMTA. A study of the influence of the test parameters on the Tg values determined and of the influence of the native structure (e.g. crystallinity) on the glass transition was also required to understand and validate the method.

\section{Materials and methods}

\section{Principles of DMTA}

The Rheometrics Solids Analyser RSAII (Rheometrics, Inc, Piscataway, NJ, USA) machine was used to compress the sample between two plates. The lower plate was moved by the actuator at a specified frequency for a specified length, thus causing an oscillatory strain input. The top plate moved to adjust a force sensor, thus measuring the resulting sinusoidal stress. 
After some time the output stress frequency is the same as the input strain frequency, but the wave is deviated by the so-called phase angle, rotating between $0^{\circ}$ for an elastic solid to $90^{\circ}$ for a pure viscous liquid. From this phase angle and the ratio of the amplitudes of the input and output sinusoids, the relationship between stress and strain is fully quantified by the following relationships:

$$
\begin{array}{ll}
\text { Input strain wave: } & \epsilon=\epsilon_{0} \sin (\omega \mathrm{t}) \\
\text { Output stress wave: } & \sigma=\sigma_{0} \sin (\omega \mathrm{t}+\delta) \\
\begin{array}{ll}
\text { Complex elastic } \\
\quad \text { modulus: }
\end{array} & \mathrm{E}^{*}=\sigma_{0} / \epsilon_{0}=\mathrm{E}^{\prime}+\mathrm{i} \mathrm{E}^{\prime \prime} \\
\text { Storage modulus: } & \mathrm{E}^{\prime}=\mathrm{E}^{*} \cos \delta \\
\text { Loss modulus: } & \mathrm{E}^{\prime \prime}=\mathrm{E}^{*} \sin \delta
\end{array}
$$

This is a typical frequency-response analysis, widely used in process dynamic studies of chemical engineering (Luyben, 1990).

$\mathrm{E}^{\prime}$ quantifies the energy that is stored by the material during the compression movement of the plates and is released back during expansion and is known as storage modulus. $E^{\prime \prime}$ quantifies the energy that is dissipated, that is, is supplied during compression but lost as thermal energy or used in molecular rearrangements and is known as loss modulus.

These relationships apply only if the material exhibits linear viscoelasticity, which implies that $\mathrm{E}^{\prime}, \mathrm{E}^{\prime \prime}$ and $\tan \delta$ must be independent of the strain amplitude. This usually requires that measurements can only be made at sufficiently low strain amplitudes. According to the frequency-response theory, $\mathrm{E}^{*}$ is also the value of the transfer function of the relationship between stress and strain in the Laplace domain, obtained by replacing the Laplace variable by wi.

In DMTA, measurements can be continuously taken while the temperature is changed inside the measuring chamber.

\section{Native flour (NF) samples}

Commercial wheat flour was used (Farinha Extreme 75, MOANOR, Porto, Portugal). The flour was equilibrated at 50\% relative humidity at $25^{\circ} \mathrm{C}$ for 2 weeks prior to measurement, corresponding to a final moisture content of $13.5 \mathrm{~g}$ of water/100g of flour (determined gravimetrically by oven drying, norm ISO 1666 1973, method II).

\section{Pre-gelatinized flour (PGF) samples}

The same commercial wheat flour (MOANOR, Porto, Portugal) was gelatinized by preparing an aqueous solution of $10 \mathrm{~g}$ of flour / $100 \mathrm{~g}$ of water and heating at $80^{\circ} \mathrm{C}$ for sufficient time to ensure full gelatinization (this was verified by DSC - Shimadzu, Japan). The resulting gel was cooled to $-28^{\circ} \mathrm{C}$ (in an air freezer) and freeze-dried at 1 psi and $20^{\circ} \mathrm{C}$ (ARMFIELD FT33, Armfield Limited, Hampshire, England). The resulting solid (moisture content $5 \mathrm{~g}$ of water $/ 100 \mathrm{~g}$ of solids) was then ground and equilibrated at $50 \%$ relative humidity at $25^{\circ} \mathrm{C}$ for 2 weeks achieving a final moisture content of $14.2 \mathrm{~g}$ of water $/ 100 \mathrm{~g}$ of solids (determined gravimetrically by oven drying, norm ISO 1666 1973, method II).

\section{Sample preparation}

Cylindrical pellets $(\phi=13 \mathrm{~mm}, \mathrm{~h}=18 \mathrm{~mm})$ were obtained by pressing the powders at 2 bar for approximately $5 \mathrm{~min}$ in a pellet press (SPECAC, Porto, Portugal Model 15.011). The pellets were immediately wrapped in aluminium foil to protect from moisture exchange.

\section{DMTA mechanical spectra measurement}

Prior to the measurements, a full cycle at all frequencies and strain amplitudes, but at room temperature, was performed in each sample, to smooth out the effect of residual compressibility in the pellets. Samples were then tested using a linear temperature increase, in the compression mode, with cylindrical plate tools (plate diameter: $13 \mathrm{~mm}$ ). The tests were conducted from ambient temperature up to $100{ }^{\circ} \mathrm{C}$. Water loss during testing was determined by weighing the initial and final samples.

The values of temperature, storage modulus $\left(E^{\prime}\right)$, loss modulus $\left(E^{\prime \prime}\right)$, viscoelastic ratio $(\tan \delta)$ and the variation of sample length $(\Delta \mathrm{L})$ were recorded during the tests by the equipment software (Rhios, version 4.44, Rheometrics, Inc, Piscataway, NJ, USA). It was not possible to repeat runs with the same sample due to significant degradation at high temperatures. Replicates were performed with different samples.

\section{Moisture loss during testing}

Without protective means, samples would dehy- 
Table 1 Experimental design for the two level factorial design for three factors

\begin{tabular}{llll}
\hline Experiment & Heating rate $\left({ }^{\circ} \mathbf{C} \mathbf{m i n}^{-1}\right)$ & Frequency $(\mathbf{H z})$ & $\begin{array}{l}\text { Strain amplitude } \\
(\times \mathbf{1 0 0 0})\end{array}$ \\
\hline 1 & 1 & 1 & 4 \\
2 & 1 & 1 & 1.3 \\
3 & 1 & 0.2 & 4 \\
4 & 1 & 0.2 & 1.3 \\
5 & 0.25 & 1 & 4 \\
6 & 0.25 & 1 & 1.3 \\
7 & 0.25 & 0.2 & 4 \\
8 & 0.25 & 0.2 & 1.3 \\
\hline
\end{tabular}

drate during measurement and therefore they were wrapped. Polyethylene film and aluminium foil were tested, comparing the weight loss during testing with the one observed with unwrapped pellets.

Different wrappings were also tested: (1) completely wrapped samples, being wrapped on the top, bottom and side; (2) partially wrapped samples, only on the side, leaving top and bottom in direct contact with the DMTA tool plates.

The samples were tested in the DMTA chamber with a temperature ramp default test, from ambient temperature to $90{ }^{\circ} \mathrm{C}$, with a strain amplitude of 0.0013 and a frequency of $1 \mathrm{~Hz}$.

\section{Heat transfer efficiency measurements}

In situ measurements of the sample temperature were made by a thermocouple (Fe-CuNi (IronConstantan) wire,) placed as close to the centre of the sample as possible, with the thermocouple end being insulated with PTFE to prevent heat conduction through the wire. Another thermocouple was placed inside the DMTA chamber to monitor chamber temperature. Both thermocouples were connected to a temperature acquisition system. Tests were then performed at different heating rates $\left(0.5\right.$ and $\left.1{ }^{\circ} \mathrm{C} \mathrm{min}-1\right)$. The frequency and strain amplitude were $1 \mathrm{~Hz}$ and 0.0013 , respectively.

\section{Measuring conditions}

To assess the influence of the test parameters on the DMTA results, native flour samples were tested using different combinations of heating rate, frequency and strain amplitude according to a replicated full factorial design at two levels.
Table 1 shows the values of the levels considered. The more significant effects were then detailed by means of a full factorial design at four levels, with three replicates, for the two input factors strain amplitude and frequency, as shown in Table 2. The heating rate was then constant and set to $1{ }^{\circ} \mathrm{C} \min ^{-1}$.

In order to assess the influence of the native structure of flour, pre-gelatinized samples were tested at five different frequencies $(0.1,0.2,0.5$, 0.8 and $1 \mathrm{~Hz}$ ), with a strain amplitude of 0.0004 and at a heating rate of $1{ }^{\circ} \mathrm{C} \mathrm{min}-1$.

\section{Results and discussion}

\section{Moisture loss}

The glass transition temperature is very sensitive to water content (Zeleznak \& Hoseney, 1987)

Table 2 Experimental design for four level factorial design for two factors

\begin{tabular}{lll}
\hline Experiment & Frequency $\mathbf{( H z )}$ & $\begin{array}{l}\text { Strain amplitude } \\
(\text { *1000) }\end{array}$ \\
\hline 1 & 0.2 & 4 \\
2 & 0.2 & 7 \\
3 & 0.2 & 10 \\
4 & 0.2 & 13 \\
5 & 0.5 & 4 \\
6 & 0.5 & 7 \\
7 & 0.5 & 10 \\
8 & 0.5 & 13 \\
9 & 0.8 & 4 \\
10 & 0.8 & 7 \\
11 & 0.8 & 10 \\
12 & 0.8 & 13 \\
13 & 1 & 4 \\
14 & 1 & 7 \\
15 & 1 & 10 \\
16 & 1 & 13 \\
\hline
\end{tabular}




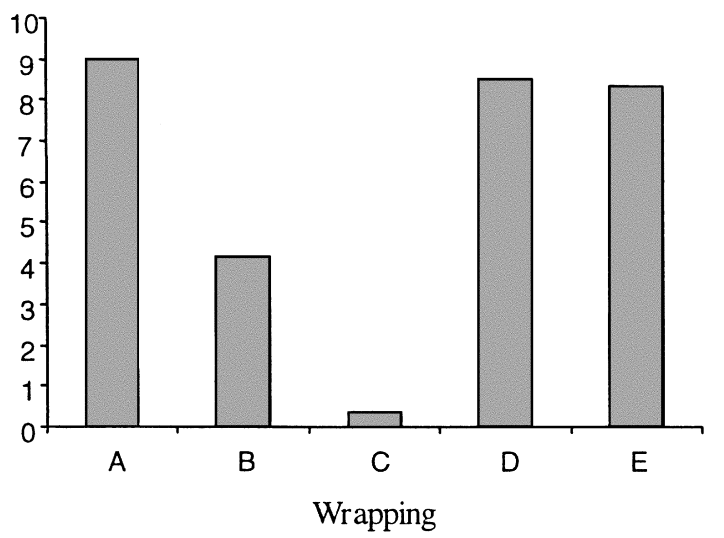

Figure 1 Moisture loss during testing. A - unwrapped sample; B - sample partially wrapped in aluminium foil; C - sample totally wrapped in aluminium foil; D sample partially wrapped in polyethilene film; E - sample totally wrapped in polyethilene film.

and though the starch was compressed in pellets that only had the sides exposed to the air in the chamber, the moisture loss during testing was too high to allow for accurate measurements, as shown in Fig. 1. However, when wrapping the samples completely in aluminium foil, moisture loss was prevented. This would not be achieved if wrapping only the sides (partial wrapping) or if using polyethylene film instead of aluminium foil, as can be seen in Fig. 1.

It must be noted that aluminium foil does not go through significant changes in the range of temperatures in question (ambient to $90^{\circ} \mathrm{C}$ ) and as the measurements are based on variations of the values of $\mathrm{E}^{\prime}$ and $\mathrm{E}^{\prime \prime}$ (that is, relative changes rather than absolute values), this procedure is acceptable.

\section{Thermal lag}

Ideally, the thermal lag between chamber and sample temperature should be negligible or constant for all test conditions, as otherwise the heating rates established in the experimental design might be slightly different from the real ones, as the thermal lag would vary somewhat with the heating rate. Moreover, the temperature gradient in the sample itself (from surface to centre temperature) might be too high and affect the desired accuracy of the readings. Figure 2 shows the thermal lag in terms of the real pellet temperature versus the chamber temperature readings. It can be seen that with full aluminium foil wrapping, the thermal lag is approximately constant, around $2{ }^{\circ} \mathrm{C}$, up to $70^{\circ} \mathrm{C}$ for the maximum heating rate used $1{ }^{\circ} \mathrm{C} \mathrm{min}^{-1}$. This is not the case of the unwrapped samples, which may be due also to the effect of the water evaporation.

\section{Mechanical spectra}

The mechanical spectra of the native flour (NF) samples showed a clear change of the viscoelastic properties according to the expected glass transition effects: a drop of $\mathrm{E}^{\prime}$ around $55^{\circ} \mathrm{C}$, a peak of
Figure 2 Thermal lag during testing for unwrapped $(\diamond)$ and wrapped $(\bigcirc)$ samples in aluminium foil.

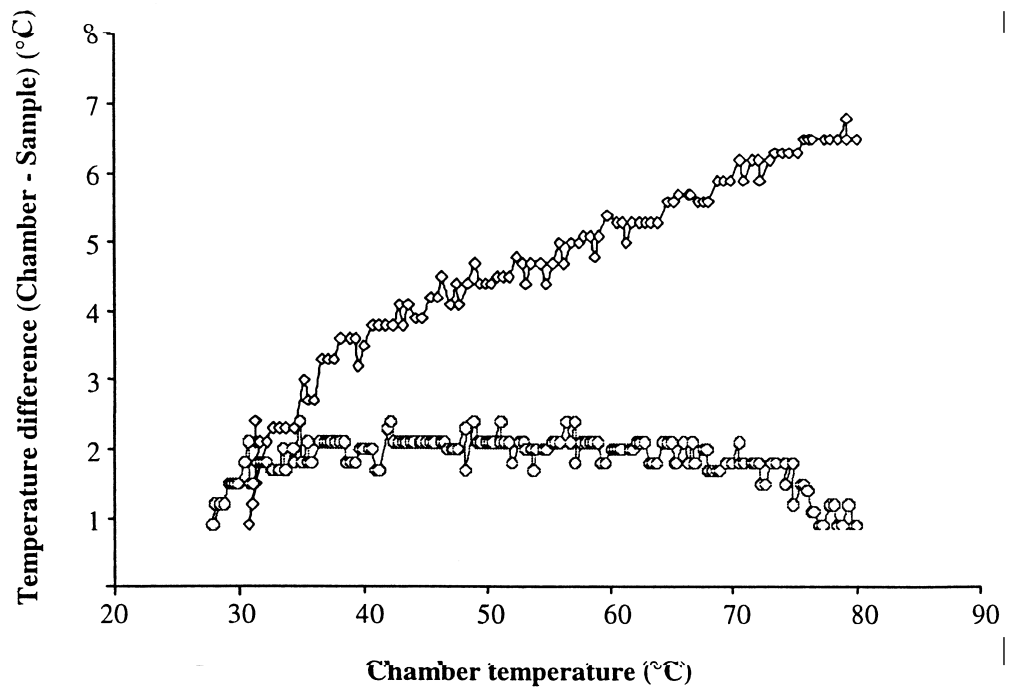


Figure 3 Mechanical spectra of native flour pellets. Frequency $0.8 \mathrm{~Hz}$; Strain amplitude: 0.0004 ; Heating rate: $1{ }^{\circ} \mathrm{C} \mathrm{min}^{-1}$.

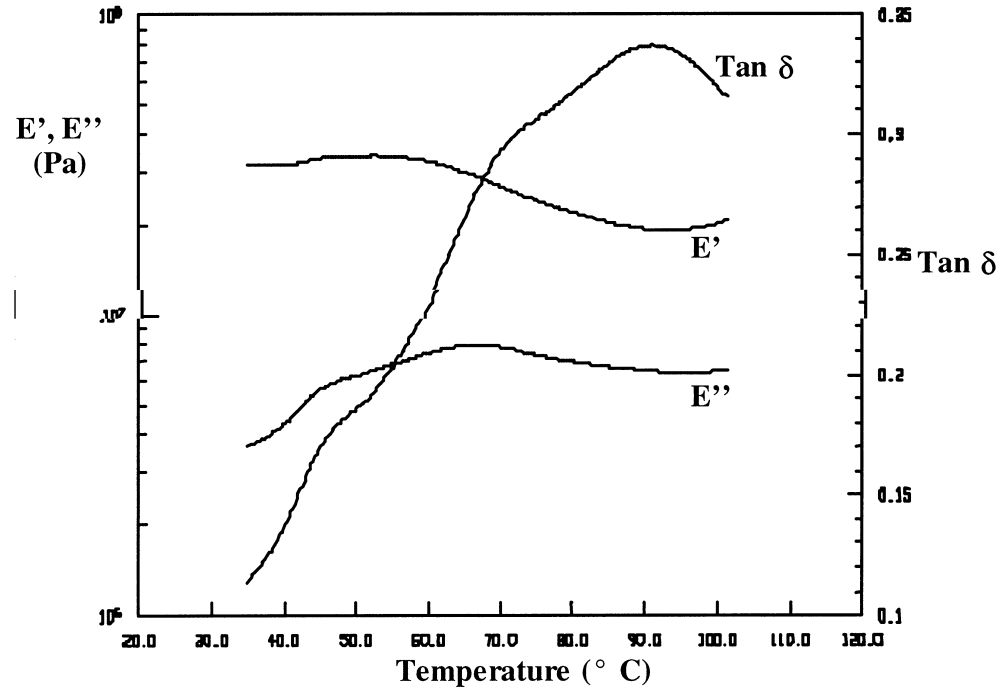

$\mathrm{E}^{\prime \prime}$ around $65^{\circ} \mathrm{C}$ and a peak of $\tan \delta$ around $85^{\circ} \mathrm{C}$. The $\mathrm{E}^{\prime \prime}$ peak was sufficiently well defined to allow for accurate measurements of its maxima. This was done by fitting the data points around the peak to a parabola and take the maximum as the maximum of this parabola, to smooth out the effect of the experimental error.

Similar patterns were found for the pre-gelatinized samples (PGF). However, while for the pregelatinized flour pellets the magnitude of the drop in $\mathrm{E}^{\prime}$ was consistent with literature data on glass transition of starchy materials (around two orders of magnitude for completely amorphous materials at this water content - the magnitude of the drop increases with water content - Kalichevsky et al., 1992), the drop in $E^{\prime}$ for the native flour sample was much lower. This was expected, as in the native flour material there are many crystalline fractions of starch that are intact and the amount of molecules that undergo the phase transition is much smaller. However, the results show that the change is sufficient for the DMTA to detect them. Typical examples are shown in Figs 3 and 4.

\section{Comparison of the results of native and pre- gelatinized flour}

The native flour samples showed a small shoulder
Figure 4 Mechanical spectra of pre-gelatinized flour pellets. Frequency $0.8 \mathrm{~Hz}$; Strain amplitude: 0.0004; Heating rate: $1{ }^{\circ} \mathrm{C} \min ^{-1}$.

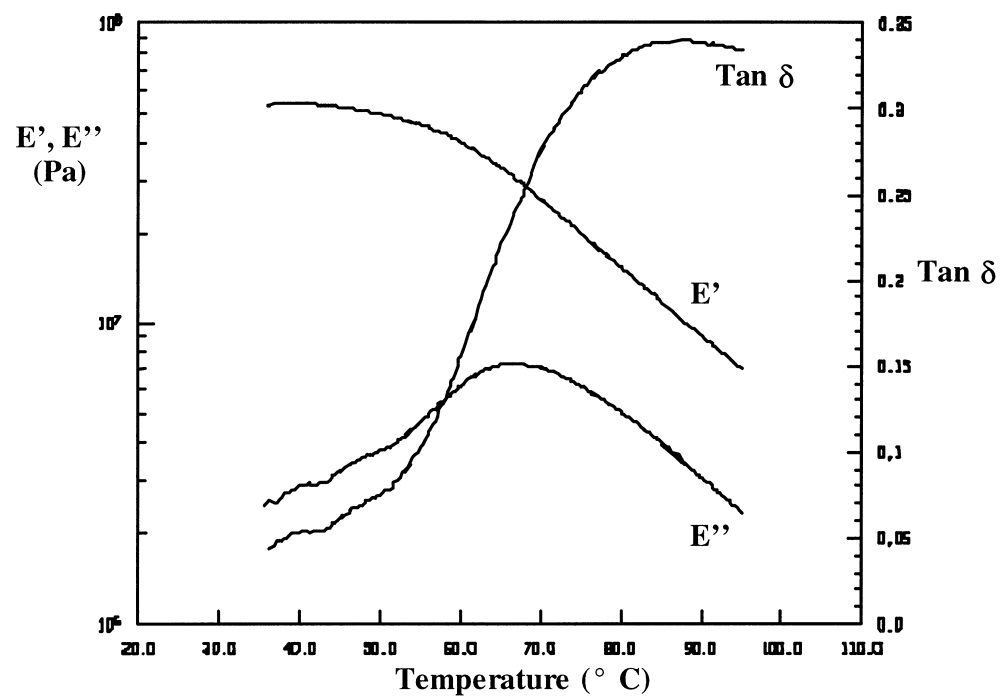


Table 3 Glass transition measurements for native (NF) and pre-gelatinised (PGF) flour pellets. Strain amplitude: 0.0004, heating rate: $1^{\circ} \mathrm{C} \mathrm{min}^{-1}$. Average and standard deviation of 3-5 replicates

\begin{tabular}{|c|c|c|c|}
\hline & $\begin{array}{l}\text { Frequency } \\
(\mathrm{Hz})\end{array}$ & $\begin{array}{l}\text { Native wheat } \\
\text { flour (NF) }\end{array}$ & $\begin{array}{l}\text { Pre-gelatinized } \\
\text { wheat flour(PGF) }\end{array}$ \\
\hline \multicolumn{4}{|l|}{ Onset } \\
\hline \multirow[t]{6}{*}{ (initial drop of $E^{\prime}$ ) } & 0.10 & $57 \pm 3$ & $54 \pm 1$ \\
\hline & 0.20 & $56 \pm 4$ & $52 \pm 5$ \\
\hline & 0.50 & $58 \pm 3$ & $58 \pm 3$ \\
\hline & 0.80 & $58 \pm 3$ & $56 \pm 1$ \\
\hline & 1.00 & $58 \pm 3$ & $57 \pm 2$ \\
\hline & & $57 \pm 3$ & $55 \pm 3$ \\
\hline \multicolumn{4}{|l|}{ Midpoint } \\
\hline \multirow[t]{6}{*}{ (E" peak) } & 0.10 & $66 \pm 4$ & $60 \pm 3$ \\
\hline & 0.20 & $65 \pm 6$ & $62 \pm 2$ \\
\hline & 0.50 & $65 \pm 2$ & $66 \pm 4$ \\
\hline & 0.80 & $69 \pm 4$ & $64 \pm 5$ \\
\hline & 1.00 & $66 \pm 2$ & $66 \pm 2$ \\
\hline & & $66 \pm 4$ & $63 \pm 3$ \\
\hline \multicolumn{4}{|l|}{ Endset } \\
\hline \multirow[t]{6}{*}{ ( $\tan \delta$ peak) } & 0.10 & $87 \pm 4$ & $70 \pm 1$ \\
\hline & 0.20 & $85 \pm 6$ & $71 \pm 4$ \\
\hline & 0.50 & $87 \pm 3$ & $74 \pm 4$ \\
\hline & 0.80 & $89 \pm 3$ & $79 \pm 6$ \\
\hline & 1.00 & $90 \pm 2$ & $76 \pm 2$ \\
\hline & & $87 \pm 4$ & $74 \pm 5$ \\
\hline
\end{tabular}

in both $\tan \delta$ and $\mathrm{E}^{\prime \prime}$ around $45^{\circ} \mathrm{C}$, which may be an indication of some other thermal transition at that temperature, not visible in the pre-gelatinized samples (this can be seen in Fig. 3 and 4). This thermal event was affected by the applied frequency and could therefore be related to the glass transition of a second component of the flour, indicating a phase separation. However, it is also possible that a small water loss could have lead to hardening and the slight increase of $\mathrm{E}^{\prime}$ around $40{ }^{\circ} \mathrm{C}$, although the moisture loss of wrapped samples is below $0.5 \%$. Wheat gluten has been considered a generally amorphous random polymer (Hoseney et al., 1986) and these shoulders may be indicating its glass transition. Although it is a small fraction of the flour (around 12\%), as in the native samples most of the starch is in the crystalline form, it is a relatively significant component of the amorphous phases of the NF samples. Phase separation between gluten and starch has already been reported in literature (Kalichevsky \& Blanshard, 1992) and is obviously significant in native flours. After gelatinizaton, this effect is no longer visible, as the thermal transition of starch now dominates the response. Furthermore, the gelatinization process may promote some mixing between starch and gluten. However, it is noted that even after cooking, starch and gluten are mostly still phase-separated.

As the samples were equilibrated to the same water activity, the pre-gelatinized flour had a slightly higher water content, due to the loss of crystallinity $(14.2 \% \mathrm{w} / \mathrm{w}$, compared to $13.5 \% \mathrm{w} / \mathrm{w}$ of the native flour pellets).

The results of the glass transition temperatures obtained are shown in Table 3 and it can be seen
Table $4 \mathrm{Tg}$ values interpolated from literature data

\begin{tabular}{llll}
\hline & \multicolumn{2}{l}{ Glass transition temperature $\left({ }^{\circ} \mathbf{C}\right)$} \\
\cline { 2 - 4 } Substance & $\begin{array}{l}\text { water content } \\
\mathbf{1 3 . 5} \%(\mathbf{w} / \mathbf{w})\end{array}$ & $\begin{array}{l}\text { water content } \\
\mathbf{1 4 . 2} \%(\mathbf{w} / \mathbf{w})\end{array}$ & Reference \\
\hline $\begin{array}{l}\text { Amylopectin } \\
\text { Starch }\end{array}$ & 72.5 & 67.2 & $\begin{array}{l}\text { Kalichevsky et al., 1992 } \\
\text { Hancock \& Zografi (1993) }\end{array}$ \\
Gluten & 58.1 & 52.7 & $\begin{array}{l}\text { Hoseny et al. 1986 } \\
\text { Kalishevski et al., 1993 }\end{array}$ \\
\hline
\end{tabular}


Figure 5 Normalized Pareto chart of the effects of test parameters on the measured $\mathrm{Tg}$ value of wheat flour. Effects for interactions that affect the response $(\mathrm{Tg})$ significantly correspond to bars that cross the 95\% line (vertical dashed line).

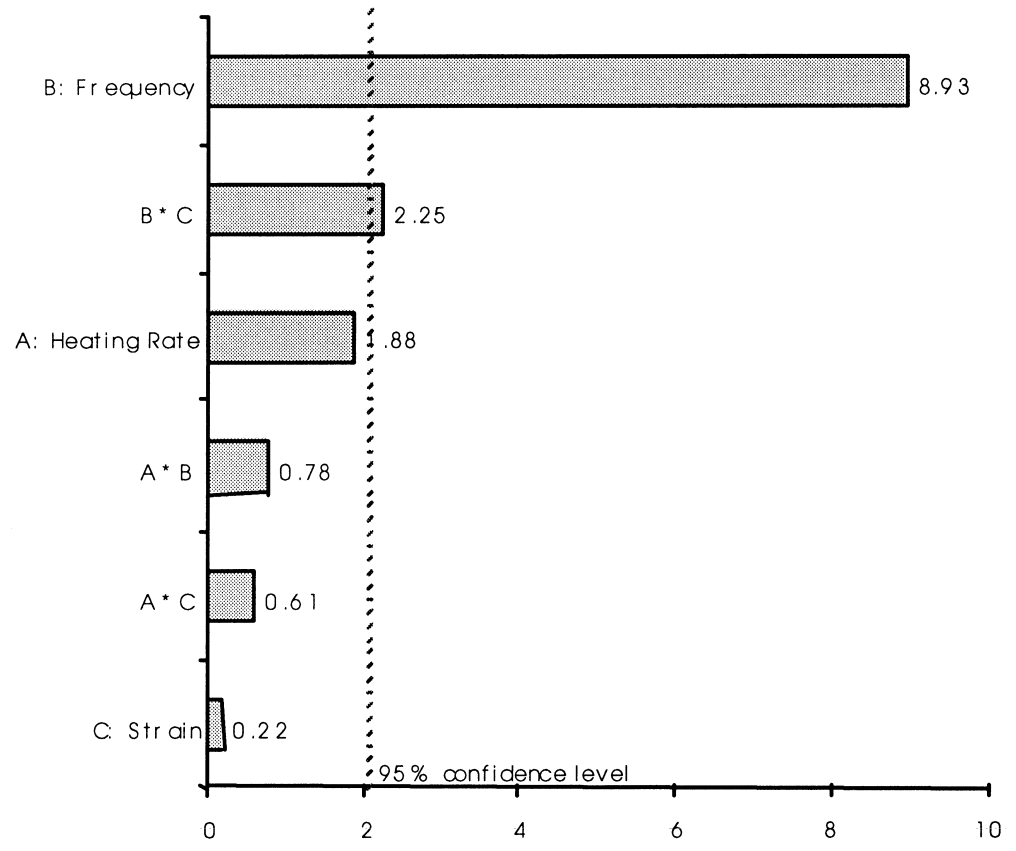

It is interesting to note that the endset of glass that the onset and midpoint of the glass transition for NF and PGF (taken as, respectively, the initial drop in $E^{\prime}$ and the peak in $E^{\prime \prime}$ ) are not statistically different at a 95\% confidence level. The values of the NF samples are slightly higher, which would agree with the fact that crystallinity has been considered to shift glass transition temperatures, as shown by the results of Zeleznak \& Hoseney, 1987 concerning measurements of gelatinized and ungelatinized wheat starch by DSC. In this case, the crystallinity is also translated by a slightly lower water content in NF then in PGF, which would justify a shift of $4-6^{\circ} \mathrm{C}$, as shown in Table 4 from literature data. The range of temperatures of the glass transition was high in both cases (between 15 and $30^{\circ} \mathrm{C}$ ), which is also in agreement with reported literature data for regions of low water content where the glass transition is broad and not very well defined (Zeleznak \& Hoseney, 1987).

The data obtained correlate well with those reported in literature for starch. The onset (drop in $\left.\mathrm{E}^{\prime}\right)$ was equal to published glass transition temperatures of starch, while the midpoint (taken as $\mathrm{Tg}$ in our work) was more close to the published $\mathrm{Tg}$ of amylopectin (see Table 3 and 4). However, it is not possible to compare different starches, because the composition of starch (and particularly the ratio amylose/amylopectin and degree of branching) greatly affects $\mathrm{Tg}$. transition (taken as the peak in $\tan \delta$ ) was significantly higher for the native flour samples. This may be due to the larger heterogeneity of the molecular arrangements in the native flour, or more likely to the difference in actual water content.

\section{Effect of the test parameters on the glass transition measurements}

As a frequency-response analysis, DMTA results are obviously influenced by the applied frequency, but it is necessary to analyze the influence of the two other major test parameters: the heating rate and the strain amplitude.

The influence of frequency can be related to the average molecular relaxation time. It can be proved that for a first-order mechanistic model, the peak of the loss modulus (maximum of the energy dissipation) corresponds to the resonance point between the applied frequency and the average molecular relaxation time (Allen, 1993). This would imply that several measurements of the $E^{\prime \prime}$ peak at different frequencies would relate to the variation of the molecular relaxation time in glass transition (Oliveira et al., 1999).

The strain amplitude should not affect the results. It must be noted that the DMTA procedure requires that measurements are taken in the 
Figure 6 Interactive effect between frequency and strain. Values presented are averages of three replicate experiments.

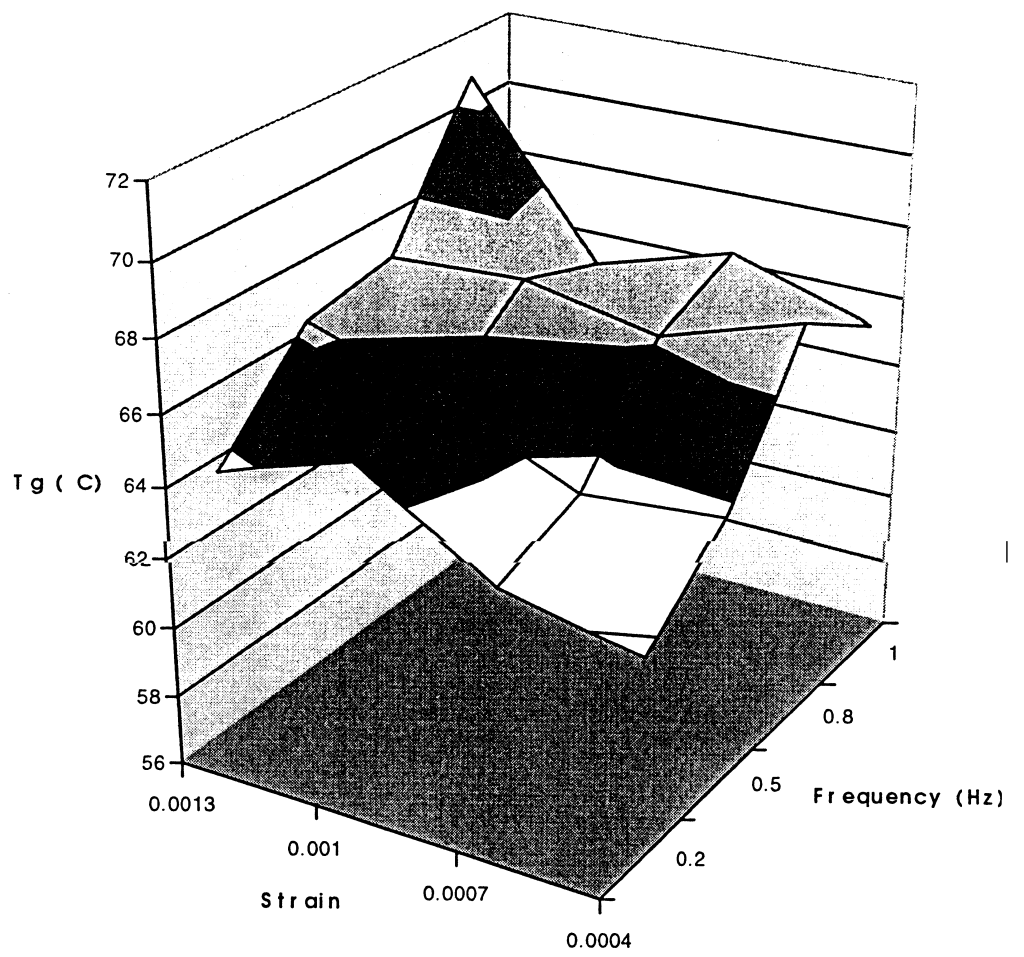

linear viscoelastic region, which must be ensured by a frequency sweep test in preliminary tests. The acceptable range of strain amplitudes is thus determined. However, the strain amplitude affects the amount of mechanical energy that is supplied to the sample and if this has a relevant effect compared to the major source of energy (heat), it could affect the results somewhat.

The heating rate would affect the results if the molecular rearrangements that are involved in the glass transition are not (relatively) instantaneous and a lag would occur between the measured temperature and the observed effects.

The first factorial design allowed for an analysis of the effects of these 3 parameters, for a reasonable range of values. Figure 5 shows the Pareto chart of the normalized effects of the factors and their interactions (a factor or interactions that have a significant effect on the response correspond to bars that cross the specified confidence level line). It can be seen that strain amplitude has no effect, indicating the linear viscoelastic behaviour, and that the heating rate range was insufficient to cause a significant variation also, at a 95\% confidence level. However, a rather curious interactive effect of strain ampli- tude and frequency was detected and to analyse this effect in more detail, the second factorial design was performed.

Figure 6 shows the response surface plot of the interactive effect of frequency and strain amplitude on the Tg measurements ( $E^{\prime \prime}$ peak). It can be seen that the interactive effect is due to a larger influence of the frequency on Tg for strain amplitudes above 0.001 and that only at the higher frequencies (above $0.8 \mathrm{~Hz}$ ) the strain amplitude has a significant effect on the results. It can be concluded that the region of high frequency and high strain amplitude should be avoided. This result is consistent with the possibility of the mechanical energy input affecting the results slightly, at these conditions, supplementing the thermal energy input and thus facilitating glass transition. It is noted that the measuring conditions were still considered to be in the linear viscoelastic range.

\section{Conclusions}

DMTA measurements of compressed pellets of native flour give adequate indication of the $\mathrm{Tg}$ of the starch, with minimal disturbance of the natural structure. The glass transition midpoint of 
the main thermal event of NF and PGF was not statistically different, but the temperature range was slightly larger in the native flour samples. Slight effects of phase separation between gluten and starch were observed in the native materials. A slight influence of the strain amplitude at high frequencies was observed, that may be due to the influence of the mechanical energy input on the sample, meaning that not only temperature but probably total energy input could play a role in this transition.

\section{References}

Allen, G. (1993). A history of the glassy state. In: The Glassy State in Foods. (eds. J. M. V. Blanshard \& P. J Lillford), Chapter 1. Nottingham, UK: Nottingham University Press.

Biliaderis, C.G., Page, C.M., Maurice, T.J. \& Juliano, B.O. (1986). Thermal characterization of rice starches: a polymeric approach to phase transitions of granular starch. Journal of Agriculture and Food Chemistry, 34, 6-14.

Graaf, E.M., Madeka, H., Cocero, A.M. \& Kokini, J.L. (1993). Determination of the effect of moisture on gliadin glass transition using mechanical spectrometry and differential scanning calorimetry. Biotechnology Progress, 9, 213-216.

Hancock, B.C. \& Zografi, G. (1993). The relationship between the glass transition temperature and the water content on amorphous pharmaceutical solids. Pharmaceutical Research, 11, 471-477.

Hoseney, R.C., Zeleznak, K. \& Lai, C.S. (1986). Wheat gluten: a glassy polymer. Cereal Chemistry, 63, 285-286.

Kalichevsky, M.T. \& Blanshard, J.M.V. (1992). A study on the effect of water on the glass transition of 1: 1 mixtures of amylopectin, casein and gluten using DSC and DMTA. Carbohydrate Polymers, 19, 271-278.

Kalichevsky, M.T. \& Blanshard, J.M.V. (1993). The effect of fructose and water on the glass transition of amylopectin. Carbohydrate Polymers, 20, 107-113.

Kalichevsky, M.T., Jaroszkiewicz, E.M., Ablett, S., Blanshard, J.M.V. \& Lillford, P.J. (1992). The glass transition of amylopectin measured by DSC, DMTA and NMR. Carbohydrate Polymers, 18, 77-88.

Kalichevsky, M.T., Jaroszkiewicz, E.M. \& Blanshard, J.M.V. (1992a). Glass transition of gluten. I. Gluten and gluten-sugar mixtures. International Journal of Biological Macromolecules, 14, 257-266.

Kalichevsky, M.T., Jaroszkiewicz, E.M. \& Blanshard, J.M.V. (1992b). Glass transition of gluten. II. The effect of lipids and emulsifiers. International Journal of Biological Macromolecules, 14, 267-273.

Luyben, W.M. (1990). Process Modeling, Simulation and Control for Chemical Engineering. 2nd edn. New York, USA: MacGraw Hill.

MacInnes, W.M. (1993). Dynamic mechanical thermal analisys of sucrose solutions. In: The Glassy State in Foods, Chapter 11. (eds. J.M.V. Blanchard \& P.J. Lillford). Nottingham, UK: Nottingham University Press.

Oliveira, J.C., Pereira, P.M., Frias, J.M., Cruz, I.B. \& MacInnes, W.M. (1999). Application of the concepts of biomaterials science to the quality optimization of frozen foods. In: Processing Foods: Quality Optiization and Process Assessment, Chapter, 7, (eds. Oliveira, F.A.R. \& Oliveira, J.C.) Boca Raton, FL, USA: CRC Press.

Peleg, M. (1994). A model of mechanical changes in biomaterials at and around their glass transition. Biotechnology Progress, 10, 385-388.

del Pilar-Buera, M., Chirife, J. \& Karel, M. (1995). A study of acid-catalyzed sucrose hydrolysis in an amorphous polymeric matrix at reduced moisture contents. Food Research International, 28, 359-365.

Roos, Y. (1995). Characterization of food polymers using state diagrams. Journal of Food Engineering, 24, 339-360.

Slade, L. \& Levine, H. (1991). Beyond Water Activity: Recent Advances Based on an Alternative Approach to the Assessment of Food Quality and Safety. Critical Reviews in Food Science and Nutrition, 30, 115-360.

Zeleznak, K.J. \& Hoseney, R.C. (1987). The glass transition in starch. Cereal Chemistry, 64, 121-124. 